\title{
EVIDENCE OF NON-THERMAL X-RAY EMISSION FROM HH 80
}

\author{
J. López-SAntiago ${ }^{1}$, C. S. Peri ${ }^{2,3}$, R. Bonito ${ }^{4,5}$, M. Miceli $^{5}$, J. F. Albacete-Colombo ${ }^{6}$, P. Benaglia $^{2,3}$, And E. De Castro $^{7}$ \\ ${ }^{1}$ Instituto de Matemática Interdisciplinar, S. D. Astronomía y Geodesia, Facultad de Ciencias Matemáticas, Universidad Complutense \\ de Madrid, E-28040 Madrid, Spain \\ ${ }^{2}$ Instituto Argentino de Radioastronomía (IAR), CCT La Plata (CONICET), C.C.5, 1894 Villa Elisa, Buenos Aires, Argentina \\ ${ }^{3}$ Facultad de Ciencias Astronómicas y Geofísicas, Universidad Nacional de La Plata, Paseo del Bosque s/n, 1900 La Plata, Argentina \\ ${ }^{4}$ Dipartimento di Fisica e Chimica, Università di Palermo, Piazza del Parlamento 1, I-90134 Palermo, Italy \\ ${ }^{5}$ INAF-Osservatorio Astronomico di Palermo, Piazza del Parlamento 1, I-90134 Palermo, Italy \\ ${ }^{6}$ Universidad Nacional del COMAHUE, Monseñor Esandi y Ayacucho, 8500 Viedma, Río Negro, Argentina \\ ${ }^{7}$ Dpto. de Astrofísica y CC. de la Atmósfera, Universidad Complutense de Madrid, E-28040 Madrid, Spain \\ Received 2013 August 22; accepted 2013 September 16; published 2013 October 3
}

\begin{abstract}
Protostellar jets appear at all stages of star formation when the accretion process is still at work. Jets travel at velocities of hundreds of $\mathrm{km} \mathrm{s}^{-1}$, creating strong shocks when interacting with the interstellar medium. Several cases of jets have been detected in X-rays, typically showing soft emission. For the first time, we report evidence of hard X-ray emission possibly related to non-thermal processes not explained by previous models of the post-shock emission predicted in the jet/ambient interaction scenario. HH 80 is located at the south head of the jet associated with the massive protostar IRAS 18162-2048. It shows soft and hard X-ray emission in regions that are spatially separated, with the soft X-ray emission region situated behind the region of hard X-ray emission. We propose a scenario for $\mathrm{HH} 80$ where soft X-ray emission is associated with thermal processes from the interaction of the jet with denser ambient matter and hard X-ray emission is produced by synchrotron radiation at the front shock.
\end{abstract}

Key words: Herbig-Haro objects - ISM: jets and outflows - radiation mechanisms: non-thermal - stars: individual (IRAS 18162-2048) - stars: pre-main sequence - X-rays: general

\section{INTRODUCTION}

Knots observed in the optical band (Herbig-Haro objects, $\mathrm{HH}$ ) originating from a young stellar object (YSO) and traveling into the surrounding medium are associated with shock fronts and post-shock regions. The HH knotty structure along the jet axis, typically observed as a chain of knots, has been interpreted as pulses in the ejection of material by the star (see the review in Bonito et al. 2010b), which can cause mutual interaction and collisions between knots (an example of a collision between optical knots is discussed in Bonito et al. 2008, for an X-ray emitting jet). Hydrodynamic models predict $\mathrm{X}$-ray emission by mechanical heating at the shock front, caused by the interaction between the jet and the ambient, with the shocked material reaching temperatures of $1 \mathrm{MK}$ (Bonito et al. 2004), thus emitting soft X-rays.

Supersonic shock fronts and post-shock regions along the jet have been detected in a wide wavelength range, from radio to optical bands (Reipurth \& Bally 2001, and references therein) and can also be detected in the UV band (Ortolani \& Dodorico 1980; Hollis et al. 1991; Liseau et al. 1996; Gómez de Castro \& Verdugo 2001; Gómez de Castro \& von Rekowski 2011; Coffey et al. 2012; Schneider et al. 2013). In recent years, several protostellar jets have also been revealed in X-rays (Pravdo et al. 2001, 2004; Favata et al. 2002, 2006; Bally et al. 2003; Kastner et al. 2005; Grosso et al. 2006; Güdel et al. 2008; Bonito et al. 2010a, 2010b, 2011).

The HH complex HH 80-81 was detected in X-rays by Pravdo et al. (2004). HH 80-81 is the southern part of an extended jet whose main source is the high-mass protostar IRAS 18162$2048\left(L=17000 L_{\odot}\right.$; Carrasco-González et al. 2010), which is situated at a distance of $1.7 \mathrm{kpc}$ (Rodríguez \& Reipurth 1989) in the direction of the Sagittarius arm. With a projected extension of $\simeq 5 \mathrm{pc}$, this is one of the largest protostellar jets ever detected (see also Tsujimoto et al. 2004; Rodríguez et al. 2005; CarrascoGonzález et al. 2010). Pravdo et al. (2004) distinguished two $\mathrm{X}$-ray sources coincident with $\mathrm{HH}$ 80: a soft X-ray source $(T \sim 1.5 \mathrm{MK}$ ) related to $\mathrm{HH} 80 \mathrm{~A}$ (the northern and brighter part of HH 80), and a hard source to the south of another $\mathrm{H}_{\alpha}$ condensation (HH 80E) that they fitted with similar accuracy to both a high-temperature thermal model and a power law with an index $\sim 1$ (for a complete list of optical knots see Heathcote et al. 1998). From the analysis of Chandra spectra, the authors suggested that the hard X-ray source may be an embedded protostar, while the soft source is a region of shocked material associated with the jet.

The HH 80-81 complex has been extensively studied at radio frequencies. Martí et al. (1993) reported intensities and spectral indices for a number of radio bands using Very Large Array (VLA) observations. The authors indicated that radio emission from $\mathrm{HH} 80$ and $\mathrm{HH} 81$ is consistent with non-thermal radiation from relativistic electrons, according to measured spectral indices. More recently, Carrasco-González et al. (2010) showed that this jet is magnetized and linearly polarized. The latter is an indication of synchrotron radiation. The strength of the magnetic field at the base of the jet determined from the analysis of Carrasco-González et al. (2010) is $B \approx 0.2 \mathrm{mG}$. Although there is no measurement of the field strength at the position of $\mathrm{HH} 80$ and $\mathrm{HH} \mathrm{81,} \mathrm{models} \mathrm{of} \mathrm{precessing} \mathrm{jets} \mathrm{for}$ IRAS 18162-2048 reproduce well the position of these two knots and those detected to the north (Masqué et al. 2012). This result has been interpreted as proof of the resemblance between the ejection mechanism in both high- and low-mass stars.

Based on the most recent results from radio wavelengths and our analysis of the X-ray properties of HH 80 detected with both $X M M$ and Chandra (see Section 2), we propose a new scenario for $\mathrm{HH} 80$ in which the soft X-ray emission is produced in a shock front caused by the interaction of the jet with the 


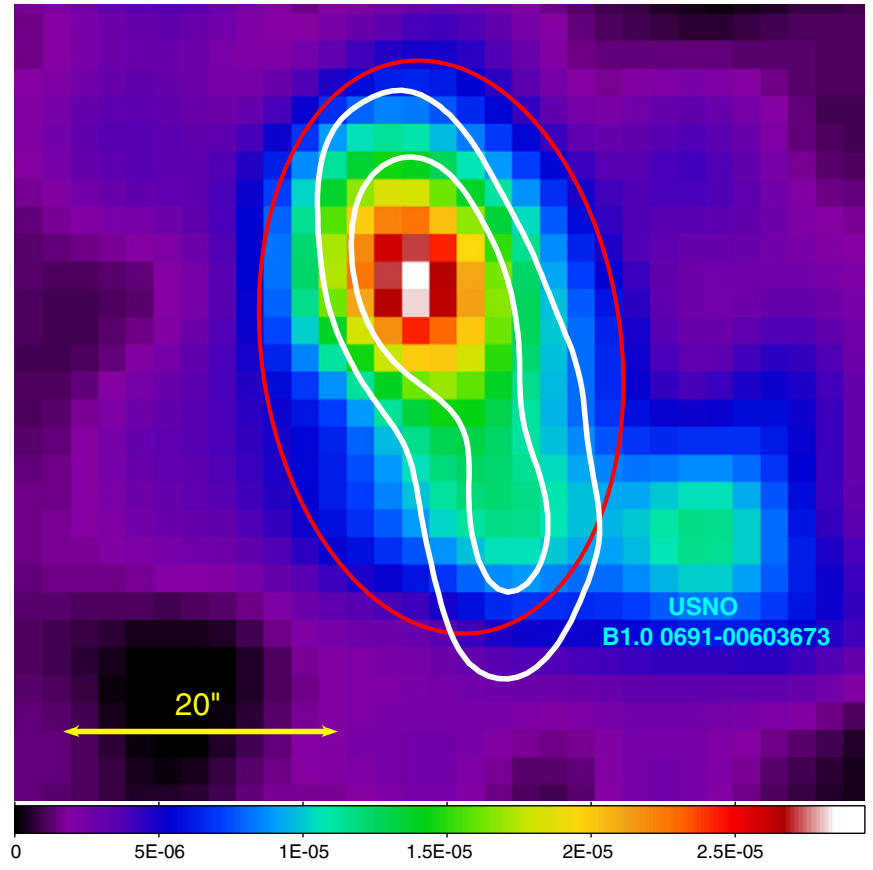

Figure 1. Count-rate image (MOS-equivalent counts per second per bin in the $0.3-1 \mathrm{keV}$ energy band). The bin size is $2^{\prime \prime}$, and the image is adaptively smoothed to an $\mathrm{S} / \mathrm{N}$ of 10 . The region selected for the spectral analysis is shown in red. Radio contours from the data presented in Martí et al. (1993) are overplotted in white. North is up and east is to the left.

ambient medium, while the hard X-ray emission is produced by synchrotron emission of electrons in the jet magnetic field. In the following sections we show a multiwavelength analysis of HH 80 that supports our hypothesis.

\section{X-RAY DATA ANALYSIS}

\subsection{XMM-Newton Observation}

We analyzed the XMM-Newton EPIC archive observation ID 0149610401 (ObsID: 2003-09-14) performed with the medium filter by using the full frame mode for the metal oxide semi-conductor (MOS; Turner et al. 2001) and pn (Strüder et al. 2001) cameras. The observation was pointed toward the central source IRAS 18162-2048. With the telescope's field-of-view of $30^{\prime}$, this observation covers the whole jet, from $\mathrm{HH} 80 \mathrm{~N}$ at the northeast to $\mathrm{HH} 80$ and $\mathrm{HH} 81$ at the southwest. We processed the data by using the Science Analysis System (SAS V12). Light curves, images, and spectra were created by selecting events with PATTERN $\leqslant 12$ for the MOS cameras, PATTERN $\leqslant 4$ for the pn camera, and FLAG $=0$ for both. To reduce the contamination by soft proton flares, the event files were screened by adopting the sigma-clipping algorithm. The screened exposure times are $29 \mathrm{ks}, 30 \mathrm{ks}$, and $23 \mathrm{ks}$ for MOS1, MOS2, and pn, respectively. Figure 1 shows the EPIC image of the source in the $0.5-1 \mathrm{keV}$ band. The image has been obtained by superimposing (with the EMOSAIC task) the MOS1, MOS2, and pn images, taking account of the differences between MOS and pn effective areas, and is background-subtracted, vignetting-corrected, and adaptively smoothed (with the task ASMOOTH).

We performed a spectral analysis in the energy band 0.3-8 keV using XSPEC V12.8. Spectra were extracted from the region shown in Figure 1 and rebinned to achieve a signal-tonoise ratio $(\mathrm{S} / \mathrm{N})$ per bin of $>4$. Spectral fittings were performed simultaneously on both MOS spectra and on the pn spectrum. The reported errors are at $90 \%$ confidence. We verified that an isothermal model of optically thin plasma (APEC model in XPSEC) cannot describe the observed spectra $\left(\chi^{2}=260\right.$ with 33 degrees of freedom; dof) and significantly underestimates the high energy part of the spectrum showing systematic residuals above $\sim 1 \mathrm{keV}$. We obtained a very good fit to the data $\left(\chi^{2}=29.1\right.$ with 31 dof) by adding a power law component to the thermal model. Figure 2 shows the pn spectrum ${ }^{8}$ with the best fit model and residuals. The thermal component, with temperature $k T_{1}=0.11 \pm 0.03 \mathrm{keV}$ describes well the low energy part of the spectrum, while contribution of the power law component, which shows a very flat spectral index $\Gamma=0.8_{-0.2}^{+0.3}$, dominates above $\sim 1.5 \mathrm{keV}$. We also adopted a model with two

8 Only the pn spectrum is shown for clarity.

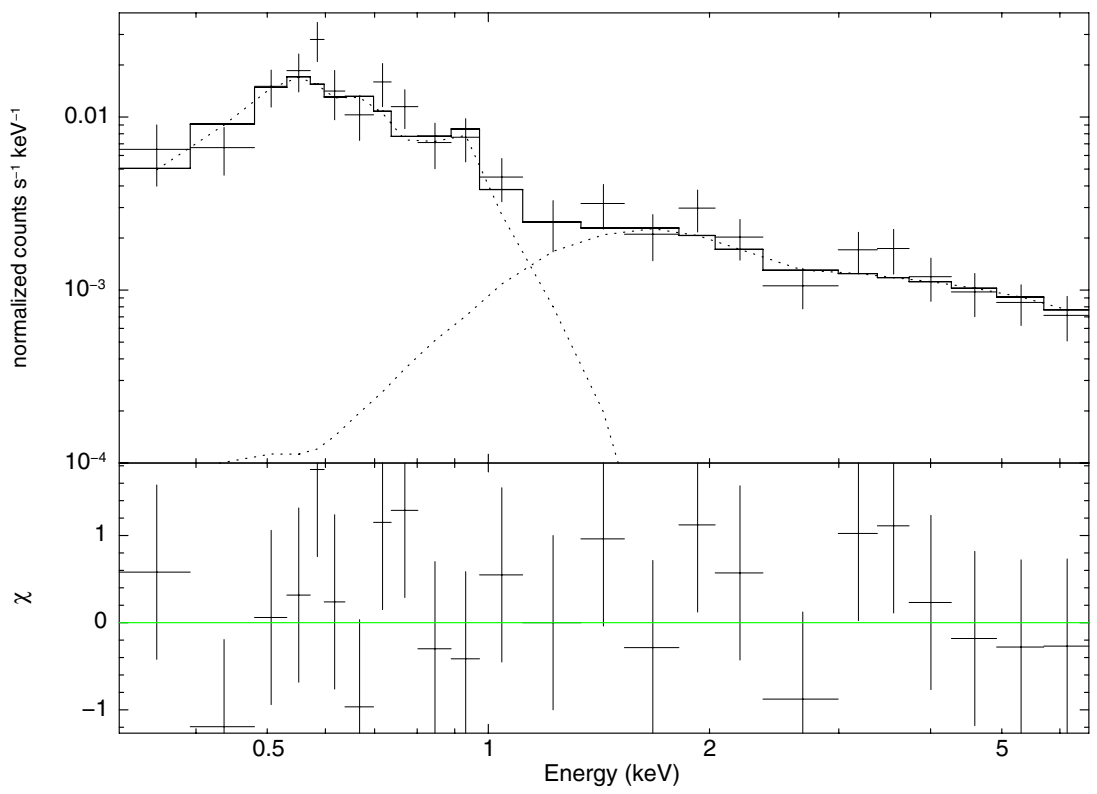

Figure 2. PN spectrum of HH 80 (extracted from the region shown in Figure 1) with the corresponding APECT + Power law best-fit model and residuals. The contribution of each component is shown. 

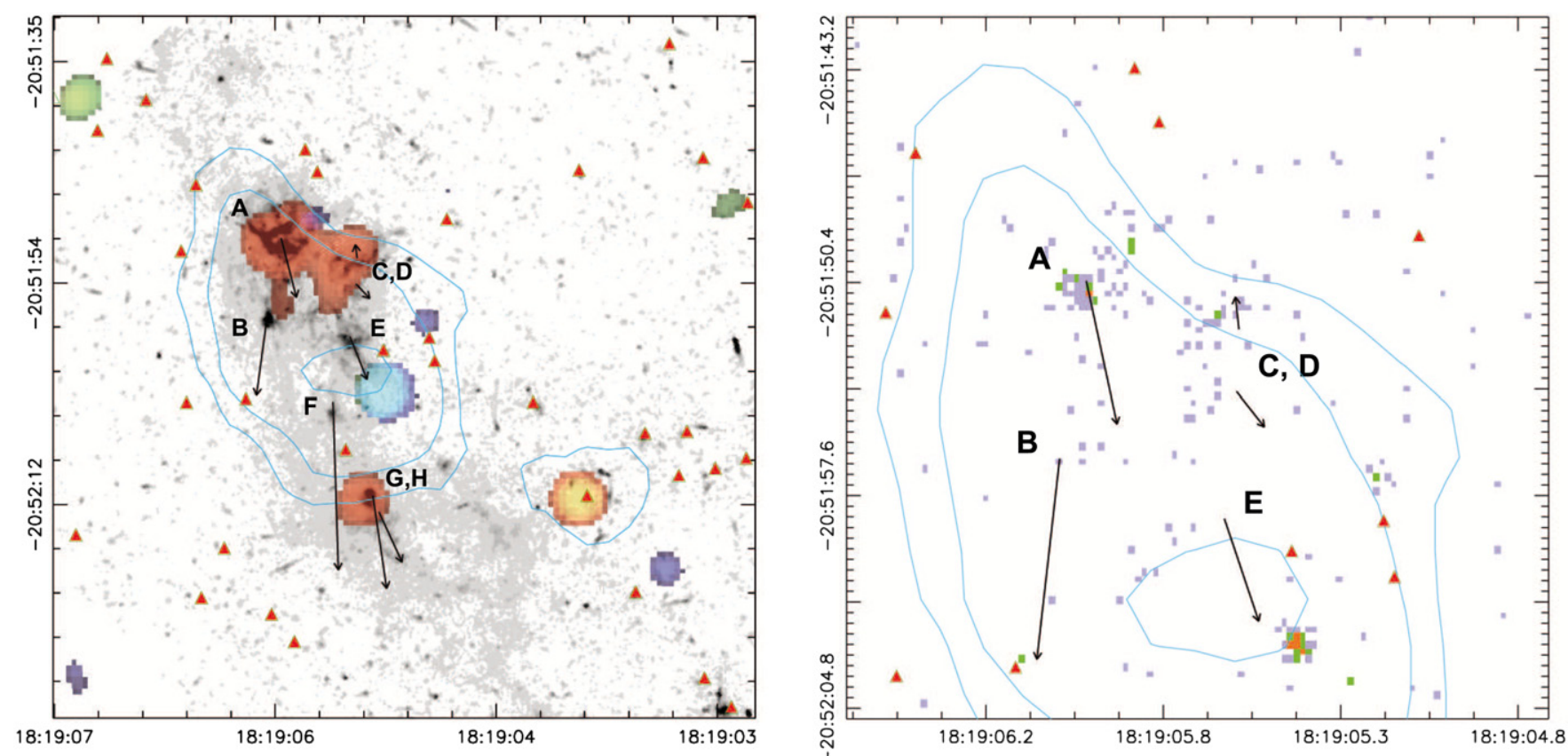

Figure 3. Left: WPC $2 \mathrm{H}_{\alpha}$ image of HH 80 with XMM-Newton EPIC-pn contours and Chandra smoothed image overplotted in false color: red for 0.3-1.2 keV, green for 1.2-2.5 keV and blue for 2.5-4.5 keV. Coordinates are J2000. Proper motions are from Heathcote et al. (1998), transformed to J2000. Filled triangles are Spitzer sources in the [3.6] $\mu \mathrm{m}$ band of IRAC with $\mathrm{S} / \mathrm{N} \geqslant 2$. Right: central region of $\mathrm{HH}$ 80. This is a refined Chandra image resulting from the sub-pixel repositioning algorithm. The binning is 0.5 . Contours, proper motions, triangles and flags are the same than in the left panel.

APEC components to investigate a possible thermal origin for the hard X-ray emission. Though this model provides an acceptable fit to the spectra $\left(\chi^{2}=33.7\right.$ with 31 dof $)$, it provides an unrealistically high value for the temperature of the hot plasma, $k T_{2}>27 \mathrm{keV}$. Therefore, we conclude that the hard X-ray emission does not have a thermal origin.

\subsection{Chandra Observation}

HH 80 was observed twice with the Advanced CCD Imaging Spectrometer (ACIS) onboard the Chandra X-ray Observatory (ObsIDs 2535 and 6405). HH 80 is off-axis in the latter, so those data were not used for our study. During the observation performed in 2002, data were acquired in faint mode for a total of $37.7 \mathrm{ks}$.

Data reduction, starting with the level 1 event list provided by the pipeline processing tool of Chandra, was carried out with CIAO 4.4 and the CALDB 4.5.3 set of calibration files. We produced a level 2 event file using the chandra_repro CIAO task, taking advantage of the F-mode enhanced background, filtering and retaining only events with grades $=0,2,3,4,6$ and status $=0$. Since the images of the ACIS-S4 chip show a variable pattern of linear streaks, we created a new bad pixel file to improve the streak detection efficiency in the S4 chip. We filtered events in the $0.5-8 \mathrm{keV}$ range. By performing a simultaneous spectral fitting of the Chandra dataset with the $X M M$ dataset, we derived compatible results as described in Section 2.1.

The Chandra/ACIS resolution allowed us to distinguish the soft and hard X-ray emitting components (see Figure 3). In order to analyze the morphology of these two components of the HH 80 complex structure, we also applied the sub-pixel repositioning algorithm available in CIAO (EDSER) to the Chandra images to refine the event positions (Li et al. 2004). The improved image can therefore be resampled at one-half of the native ACIS pixel scale $\left(00^{\prime} 5 / 2^{\prime \prime}\right)$. Both the soft and the hard components show a hint of elongation which appears, if any, aligned with the jet axis and the proper motion of the E knot (see Figure 3, right panel). Note that an asymmetry in the Chandra point-spread function (PSF) has recently been discovered located at position angle P.A. = 195-roll angle $\pm 25 \mathrm{deg}$, corresponding to P.A. $=285.844 \mathrm{deg}$ which is not coincident with the direction of the extension of the X-ray sources nor the jet axis.

\section{SEARCH FOR OPTICAL AND INFRARED COUNTERPARTS}

We looked for counterparts to the different X-ray sources associated with $\mathrm{HH} 80$ at other wavelengths, with the aim of discarding an extragalactic origin for the X-ray emission in this region. We analyzed data from the Spitzer mission and inspected the $\mathrm{H}_{\alpha}$ image taken with the WFPC2 onboard the Hubble Space Telescope (Heathcote et al. 1998). From Figure 3, it seems clear that there is no point-like source associated with the hard X-ray source (below knot E), while the soft X-ray sources are related to different $\mathrm{H}_{\alpha}$ knots of the jet (precisely knots A-D).

The Spitzer Heritage Archive at the NASA, IPAC Infrared Science Archive contains one observation from the GLIMPSE project covering the region of HH 80-81 (AORKEY 21289984) and a pointed observation in map mode with both the Infrared Array Camera (IRAC; AORKEY 11069952) and the Multiband Imaging Photometry for SIRTF (MIPS; AORKEY 11072256). With a configuration with frame time $10.4 \mathrm{~s}$, the pointed observation of $\mathrm{HH} 80-81$ is substantially deeper, reaching limiting magnitudes in the [3.6] $\mu \mathrm{m}$ band $\simeq 20$ mag for sources detected above $\mathrm{S} / \mathrm{N}=2(F \simeq 30 \mu \mathrm{Jy})$. We followed the procedure described in López-García et al. (2013) to find sources in this Spitzer observation and performed aperture photometry. Both panels in Figure 3 show the infrared sources detected in 
the proximities of $\mathrm{HH} 80$ (filled triangles). The only infrared source close to the hard X-ray source is located $>5^{\prime \prime}$ to the north of the Chandra source and it is detected only in the [3.6] $\mu \mathrm{m}$ band with $\mathrm{S} / \mathrm{N} \simeq 2$. Given this low IR-signal and the size of the angular offset, any physical association is unlikely.

\section{DISCUSSION}

HH $80-81$ is the head of a jet whose origin is the massive protostar IRAS 18162-2048. The jet emits at radio frequencies (Martí et al. 1993) and this emission is linearly polarized (Carrasco-González et al. 2010). The region of HH 80 shows radio emission with a negative spectral index $\alpha=-0.3$ (Martí et al. 1993), indicating that it is dominated by non-thermal radiation (e.g., Ginzburg \& Syrovatskii 1964). In addition to HH 80-81, only a few YSOs show evidence of non-thermal radio emission in their outflows: Serpens triple radio source, Cepheus A East sources 1-7, W3 $\left(\mathrm{H}_{2} \mathrm{O}\right)$, L778 VLA 5 and IRAS 16547-4247 (see Carrasco-González et al. 2010, and references therein). Radio-synchrotron polarized emission has been detected only in IRAS 18162-2048.

The HH 80 XMM-Newton spectrum shows a soft component, which is compatible with thermal plasma emission at a temperature of $\sim 10^{6} \mathrm{~K}$, plus a hard component with a power law spectrum with the index $\alpha \simeq 0.8$. Chandra is able to separate both spectral components spatially. The soft component is correlated with the denser $\mathrm{H}_{\alpha}$ region ( $\mathrm{HH} 80 \mathrm{~A}, \mathrm{C}$ and $\left.\mathrm{D}\right)$. The hard component is located just in front of $\mathrm{HH} 80 \mathrm{E}$ in a region with low gas density (see Figure 3).

The results of Bonito et al. (2010a) suggest that $\mathrm{HH} \mathrm{80/81} \mathrm{and}$ probably also the X-ray emitting jet discovered by Tsujimoto et al. (2004; TKH8), for which high velocities are needed to explain their spectra, are not compatible with the other soft $\mathrm{X}$-ray emitting jets well modeled by the post-shock emission due to the jet/ambient interaction (as in the wide exploration of the parameter space also performed in Bonito et al. 2007). In Figure 7 of Bonito et al. (2010a), in fact, both HH 80 and TKH8 cannot be described by the model of the emission from the post-shock formed by the jet/ambient interaction as in the cases of the other jets emitting soft X-rays; furthermore, the shock velocity deduced in these two jets is high with respect to the other soft jets.

The analysis of the morphology of the Chandra/ACIS detected source at sub-pixel resolution shows a hint of elongation of the hard component along the jet axis and the proper motion of the E knot (note that the PSF asymmetry has been computed and is not aligned with the jet axis but, on the contrary, it is almost perpendicular to the proper motion of the knots within the jet). Available Chandra/ACIS data allowed us to discriminate the two X-ray emitting components detected in $\mathrm{HH}$ 80: the soft component is almost coincident with the A knot, and the hard component is located along the jet axis further away with respect to the E knot and along its proper motion (see Figure 3, right panel). A deeper Chandra observation could help in distinguishing the hard component morphology and in performing a spatially resolved spectral analysis of the different sources. Also with a time baseline of more than $10 \mathrm{yr}$, it will be possible to measure the proper motion of the source, if any, and compare with radio proper motions.

The hard X-ray source has been previously proposed to be an embedded protostar not related to the jet itself (see Pravdo et al. 2004). To explore this scenario, we looked for counterparts to the hard X-ray source in any photometric band, from optical to infrared. As we mentioned in Section 3, no counterpart was found down to $20 \mathrm{mag}$ in the Spitzer/IRAC bands or in MIPS data. This result likely discards the scenario of the embedded protostar.

The possible relation of the hard X-ray source with a highly obscured active galactic nucleus (AGN) appears also unlikely. With a measured (unabsorbed) X-ray flux in the [0.3-10] keV

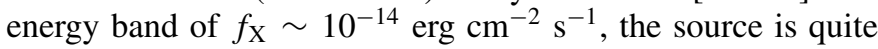
bright in X-rays. According to the $\log N-\log S$ distribution of bright AGNs (e.g., Della Ceca et al. 2004), the density of these objects at this flux is $\sim 100 \mathrm{deg}^{-2}$. Following Combi et al. (2011), the probability that an AGN was detected inside the $X M M$-Newton contours is $P \sim 0.15(15 \%)$ and the probability that it was situated at less than $5^{\prime \prime}$ of the hard X-ray source is $P<0.02(2 \%)$.

At a distance of $1.7 \mathrm{kpc}$, the X-ray luminosity of the hard Xray source is $L_{\mathrm{X}} \simeq 3 \times 10^{30} \mathrm{erg} \mathrm{s}^{-1}\left(f_{\mathrm{X}} \sim 10^{-14} \mathrm{erg} \mathrm{cm}^{-2} \mathrm{~s}^{-1}\right.$ for the hard X-ray source). This is very close to the value expected by Bosch-Ramon et al. (2010) for secondary electrons and positrons from proton-proton collisions (see their Figure 5 and Araudo \& Rodríguez 2012). Note that the authors considered a particle density $n_{c} \sim 10^{2}-10^{3} \mathrm{~cm}^{-3}$, bolometric luminosity $L_{\star} \simeq 2 \times 10^{4} L_{\odot}$ and jet velocity at the base $v_{j} \sim 10^{3} \mathrm{~km} \mathrm{~s}^{-1}$ (see Table 1 of Bosch-Ramon et al. 2010, for a complete list of observed and derived parameters for $\mathrm{HH}$ 80). The magnetic field determined by the authors at the position of $\mathrm{HH} 80$ is $B \sim 0.02 \mathrm{mG}$. This is one order of magnitude lower than the magnetic field strength determined by Carrasco-González et al. (2010) at the base of the jet but consistent with the value expected at the distance of $\mathrm{HH} 80$ from the star (Bosch-Ramon et al. 2010).

\section{CONCLUSIONS}

Supported by the results of our analysis of X-ray Chandra and XMM-Newton data and cross-correlation with optical and infrared databases, we propose a new scenario for the nature of the hard X-ray emission detected at the edge of HH 80. While the soft X-ray emission is produced in the post-shock region after interaction of the jet with dense medium, the hard X-ray emission is produced by synchrotron radiation of accelerated particles at the front-shock. The spectral index of the power law spectrum in both X-ray and radio wavelengths is consistent with this hypothesis. In addition, the X-ray luminosity assuming a distance of $1.7 \mathrm{kpc}$ agrees well with the predictions by models of non-thermal emission for HH 80. This is the first evidence of non-thermal emission detected in X-rays for a stellar jet.

This work was supported by the Spanish Government (AYA2011-29754-C03-01 and AYA2011-29754-C03-03). C.S.P. was supported by the COSPAR Capacity Building Workshop Fellowship Program and the Universidad Nacional de La Plata to develop this work at the Universidad Complutense de Madrid. M.M. is supported through the ASI-INAF contract I/009/10/0. JFAC is a staff researcher of the CONICET and acknowledges support by grant PIP 2011 0100285. J.L.-S. would like to thank Drs. F. J. Carrera, P. G. Pérez-González and S. Mateos for fruitful discussion on the spectral X-ray emission properties of AGN. We also acknowledges the referee of this manuscript for their suggestions and comments.

Facilities: XMM-Newton (EPIC), Chandra (ACIS), Spitzer (IRAC, MIPS) 


\section{REFERENCES}

Araudo, A. T., \& Rodríguez, L. F. 2012, in AIP Conf. Ser. 1505, High Energy Gamma-Ray Astronomy, ed. F. A. Aharonian, W. Hofmann, \& F. M. Rieger (Melville, NY: AIP), 281

Bally, J., Feigelson, E., \& Reipurth, B. 2003, ApJ, 584, 843

Bonito, R., Fridlund, C. V. M., Favata, F., et al. 2008, A\&A, 484, 389

Bonito, R., Orlando, S., Miceli, M., et al. 2010a, A\&A, 517, A68

Bonito, R., Orlando, S., Miceli, M., et al. 2011, ApJ, 737, 54

Bonito, R., Orlando, S., Peres, G., Favata, F., \& Rosner, R. 2004, A\&A, 424, L1

Bonito, R., Orlando, S., Peres, G., Favata, F., \& Rosner, R. 2007, A\&A, 462,645

Bonito, R., Orlando, S., Peres, G., et al. 2010b, A\&A, 511, A42

Bosch-Ramon, V., Romero, G. E., Araudo, A. T., \& Paredes, J. M. 2010, A\&A, 511, A8

Carrasco-González, C., Rodríguez, L. F., Anglada, G., et al. 2010, Sci, 330, 1209

Coffey, D., Rigliaco, E., Bacciotti, F., Ray, T. P., \& Eislöffel, J. 2012, ApJ, 749, 139

Combi, J. A., Albacete Colombo, J. F., Pellizza, L., et al. 2011, Ap\&SS, 331, 53

Della Ceca, R., Maccacaro, T., Caccianiga, A., et al. 2004, A\&A, 428, 383

Favata, F., Bonito, R., Micela, G., et al. 2006, A\&A, 450, L17

Favata, F., Fridlund, C. V. M., Micela, G., Sciortino, S., \& Kaas, A. A. 2002, A\&A, 386, 204

Ginzburg, V. L., \& Syrovatskii, S. I. 1964, The Origin of Cosmic Rays (New York: Macmillan)
Gómez de Castro, A. I., \& Verdugo, E. 2001, ApJ, 548, 976

Gómez de Castro, A. I., \& von Rekowski, B. 2011, MNRAS, 411, 849

Grosso, N., Feigelson, E. D., Getman, K. V., et al. 2006, A\&A, 448, L29

Güdel, M., Skinner, S. L., Audard, M., Briggs, K. R., \& Cabrit, S. 2008, A\&A, 478,797

Heathcote, S., Reipurth, B., \& Raga, A. C. 1998, AJ, 116, 1940

Hollis, J. M., Oliversen, R. J., Michalitsianos, A. G., Kafatos, M., \& Wagner, R. M. 1991, ApJ, 377, 227

Kastner, J. H., Franz, G., Grosso, N., et al. 2005, ApJS, 160, 511

Li, J., Kastner, J. H., Prigozhin, G. Y., et al. 2004, ApJ, 610, 1204

Liseau, R., Huldtgren, M., Fridlund, C. V. M., \& Cameron, M. 1996, A\&A, 306,255

López-García, M. A., López-Santiago, J., Albacete-Colombo, J. F., PérezGonzález, P. G., \& de Castro, E. 2013, MNRAS, 429, 775

Martí, J., Rodríguez, L. F., \& Reipurth, B. 1993, ApJ, 416, 208

Masqué, J. M., Girart, J. M., Estalella, R., Rodríguez, L. F., \& Beltrán, M. T. 2012, ApJ, 758, L10

Ortolani, S., \& Dodorico, S. 1980, A\&A, 83, L8

Pravdo, S. H., Feigelson, E. D., Garmire, G., et al. 2001, Natur, 413, 708

Pravdo, S. H., Tsuboi, Y., \& Maeda, Y. 2004, ApJ, 605, 259

Reipurth, B., \& Bally, J. 2001, ARA\&A, 39, 403

Rodríguez, L. F., Garay, G., Brooks, K. J., \& Mardones, D. 2005, ApJ, 626, 953

Rodríguez, L. F., \& Reipurth, B. 1989, RMxAA, 17, 59

Schneider, P. C., Eislöffel, J., Güdel, M., et al. 2013, A\&A, 550, L1

Strüder, L., Briel, U., Dennerl, K., et al. 2001, A\&A, 365, L18

Tsujimoto, M., Koyama, K., Kobayashi, N., et al. 2004, PASJ, 56, 341

Turner, M. J. L., Abbey, A., Arnaud, M., et al. 2001, A\&A, 365, L27 